\title{
Upregulation of microRNA-143 reverses drug resistance in human breast cancer cells via inhibition of cytokine-induced apoptosis inhibitor 1
}

\author{
JING-HAO WANG ${ }^{1}$, XIU-WEN WANG ${ }^{2}$, DI QU $^{2}$, JI-WEN SUN $^{2}$, FEI-XIAO GUO ${ }^{2}$ and DAN LU ${ }^{2}$ \\ Departments of ${ }^{1}$ Pharmacy and ${ }^{2}$ Oncology, The Second Affiliated Hospital, \\ Harbin Medical University, Harbin, Heilongjiang 150086, P.R. China
}

Received May 2, 2016; Accepted April 6, 2017

DOI: $10.3892 / \mathrm{ol} .2017 .6078$

\begin{abstract}
Cytokine-induced apoptosis inhibitor 1 (CIAPIN1), originally termed anamorsin, is an anti-apoptotic molecule that acts as a downstream effector of the receptor tyrosine kinase-Ras signaling pathway. Overexpression of CIAPIN1 contributes to multidrug resistance (MDR) and microRNA (miR)-143 is typically considered a tumor suppressor in breast cancer. The present study aimed to evaluate the therapeutic potential of miR-143 as a treatment for drug-resistant breast cancer via the downregulation of CIAPIN1 in vitro. The expression levels of miR-143 were measured using quantitative polymerase chain reaction and the expression levels of CIAPIN1 were detected via western blot analysis. Bioinformatic analyses was additionally conducted to search for miR-143, which may potentially target CIAPIN1. Luciferase reporter plasmids were created and used to verify direct targeting. In addition, Taxol-induced drug-resistant (TDR) breast cancer cell proliferation was evaluated using the Cell Counting Kit-8 assay in vitro. The present study identified an inverse association between miR-143 and CIAPIN1 protein expression levels in breast cancer MCF-7, MDA-MB-231 and MDA-MB-453 TDR cells. Specific targeting sites for miR-143 in the 3'-untranslated region of the CIAPIN1 gene were identified, which exhibit the ability to regulate CIAPIN1 expression. It was revealed that the repression of CIAPIN1 via miR-143 suppressed the proliferation of breast cancer TDR cells. The findings of the present study verified the role of miR-143 as a tumor suppressor in breast cancer MDR via inhibition of CIAPINI translation.
\end{abstract}

\section{Introduction}

In women, breast cancer is the most common cancer type, accounting for $22.9 \%$ of all cancer cases. In China, the

Correspondence to: Dr Dan Lu, Department of Oncology, The Second Affiliated Hospital, Harbin Medical University, 246 Xuefu Road, Nangang, Harbin, Heilongjiang 150086, P.R. China

E-mail: huangwei104@126.com

Key words: microRNA-143, cytokine-induced apoptosis inhibitor 1, breast cancer incidence of breast cancer has been on the rise (1). The disease is the leading cause of mortality in patients with distant metastasis, and chemoradiotherapy for advanced breast cancer is the most effective treatment method $(2,3)$. As chemotherapy is extremely important for the treatment of breast cancer, multidrug resistance (MDR) is likely to be a major obstacle (4). MDR is a complex process involving multiple mechanisms, including the overexpression of energy-dependent transporters, which shuttle anticancer drugs into and out of cells $(5,6)$.

Cytokine-induced apoptosis inhibitor 1 (CIAPIN1) is a newly identified anti-apoptotic protein; it shares no homology with Bcl-2, caspase, the IAP family or signal-transduction molecules that regulate apoptosis (7). CIAPIN1 is associated with MDR in SGC7901/Adr gastric cancer cells $(8,9)$ and HL-60/Adr leukemia cells (10), in which CIAPIN1 upregulates P-glycoprotein (MDR1) expression (8-10), leading to drug resistance. In a study examining MCF-7/ADM breast cancer cells, the same MDR mechanism was reported (11). When RNA interference (RNAi) was used to downregulate the expression of CIAPIN1 in MCF-7/ADM breast cancer cells, a significant reduction in drug resistance was observed and MDR1 expression was inhibited (11). Therefore, we hypothesize that the CIAPINI gene is a putative target for the treatment of breast cancer MDR $(11,12)$.

Over the last 10 years, a novel class of small RNA molecules known as microRNAs (miRNAs/miRs) has been recognized as an important regulator of the initiation and progression of human cancer types, including breast cancer $(13,14)$. miR-143 is generally considered to be a tumor suppressor in breast cancer $(15,16)$, however, the mechanisms by which it is downregulated here are not yet understood. Although dysregulation of CIAPIN1 and miR-143 is associated with tumorigenesis in human breast cancer, little is known about how miR-143 acts on CIAPIN1. The current study examined whether there are direct interactions between CIAPIN1, miR-143 and the reversal of MDR in breast cancer in vitro.

\section{Materials and methods}

Cell lines. Human breast carcinoma MCF-7, MDA-MB-231, and MDA-MB-453 cell lines, as well as HEK-293 cells, were purchased from the Shanghai Institute of Cell Biology, Chinese 
Academy of Sciences (Shanghai, China). The cells were cultured in Dulbecco's modified Eagle's medium (DMEM; Invitrogen; Thermo Fisher Scientific, Inc., Waltham, MA, USA) in a $5 \% \mathrm{CO}_{2}$ incubator at $37^{\circ} \mathrm{C}$, supplemented with $10 \%$ fetal bovine serum (Gibco; Thermo Fisher Scientific, Inc.) and antibiotics $(100 \mu \mathrm{g} / \mathrm{ml}$ streptomycin and $100 \mathrm{U} / \mathrm{ml}$ penicillin).

Detection of half maximal inhibitory concentration $\left(I C_{50}\right)$ values of cells against Taxol. Each group of cells (MCF7, MDA-MB-231 and MDA-MB-453), while in the logarithmic phase, was seeded $(\sim 5,000$ cells/well $)$ in culture plates of 96 wells at $37^{\circ} \mathrm{C}$, with $5 \% \mathrm{CO}_{2}$, for $24 \mathrm{~h}$. The culture DMEM was removed when the cells adhered to the plate wall. The cells were then incubated in $100 \mu \mathrm{l}$ of medium with Taxol (catalog no. 33069-62-4; Sigma) in different doses at $37^{\circ} \mathrm{C}$. The initial concentration of Taxol was $0.01 \mu \mathrm{g} / \mathrm{ml}$, and the drug concentration was increased by 1 -fold after 3 passages of culture in vitro. The concentration of Taxol was as follows: $0.01,0.02,0.04,0.08,0.16,0.32,0.64,1.28,2.56,5.12,10.24$, 20.48 and $40.96 \mu \mathrm{g} / \mathrm{ml}$. Optical density (OD) values of the cells were tested using the CCK- 8 method after $48 \mathrm{~h}$ of incubation. The cells were then treated with $10 \mu \mathrm{l} \mathrm{CCK}-8$ reagent for $4 \mathrm{~h}$, and the CCK- 8 Cell Proliferation kit (Beyotime Institute of Biotechnology, Nantong, China) was used to measure the cell viability according to the manufacturer's instructions. The absorbance was measured at $450 \mathrm{~nm}$. The cell growth inhibition ratio $=(1-$ mean OD value of experimental group / mean OD value of control group) $x$ 100. The dose response curve was drawn in different concentrations to calculate the $\mathrm{IC}_{50}$ using a probit regression model. Each concentration, including 5 duplicate wells, was experimentally used 3 times independently to obtain a mean $\mathrm{IC}_{50}$ value.

Recombinant lentivirus generation and lentivirus infection. Lentivirus Package plasmids mix (System Biosciences, Palo Alto, CA, USA) and selected LV-miR-143 (GenePharma, Shanghai, China) were cotransfected into the MCF-7, MDA-MB-231 and MDA-MB-453 Taxol-induced drug-resistant (TDR) breast cancer cells to package and produce lentiviral vector and viral titer using the gradient dilution method. Lentivirus $\left(1 \times 10^{4} \mathrm{IFU} / \mu \mathrm{l}, 20 \mu \mathrm{l}\right)$ packaging of green fluorescent protein (GFP) was infected into the MCF-7, MDA-MB-231 and MDA-MB-453 TDR cells at various volumes to obtain the best MOI value corresponding to a concentration at which no virus toxicity effect on the cells was observed. Subsequently, viruses were added at an MOI of 20 to the experimental and control groups, which were then incubated under standard conditions. At $96 \mathrm{~h}$ after infection, cells that had the strongest GFP brightness were selected from the two wells and intermediate clone culturing was performed in the experimental group.

Luciferase reporter assay. The cDNA sequence of CIAPIN1 (NM_020313.2) was obtained from Genbank (https://www.ncbi.nlm.nih.gov/genbank/). The entire 3'-untranslated region (3'-UTR) of human CIAPIN1 was amplified using polymerase chain reaction (PCR) with human genomic DNA as a template. The PCR products were inserted into the p-MIR-reporter plasmid (Ambion, Austin, TX, USA). The correct insertion was confirmed by DNA sequencing. To test the binding specificity, the sequences that interacted with the

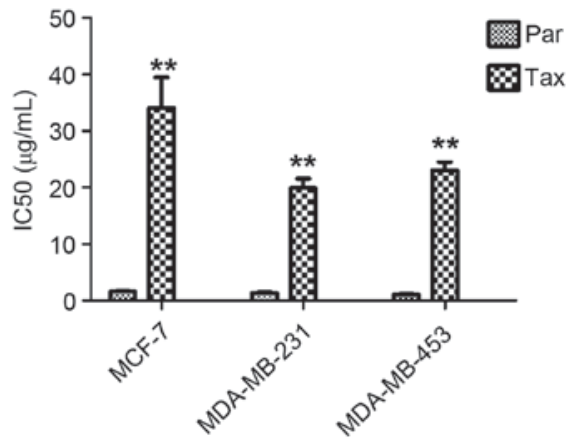

Figure 1. Taxol induces multidrug resistance. $\mathrm{IC}_{50}$ was significantly increased by Taxol in breast cancer cells. ${ }^{* *} \mathrm{P}<0.01$ for MCF7/Tax versus MCF7, MDA-MB-231/Tax versus MDA-MB-231, and MDA-MB-453/Tax versus MDA-MB-453. IC $_{50}$, half maximal inhibitory concentration Tax, Taxol; Par, non-multidrug resistant breast cancer cells or normal breast cancer cells.

seed sequence of miR-143 were mutated (from TCATCTCA to AATCCTCT for the miR-143 binding site), and the mutant (MT) CIAPIN1 3'-UTR was inserted into an equivalent luciferase reporter plasmid. For luciferase reporter assays, HEK-293 cells were seeded in 6-well plates and co-transfected with $2 \mu \mathrm{g}$ firefly luciferase reporter plasmid (pGL, pGL3-promoter vector; E1761; Promega), $2 \mu \mathrm{g} \beta$-galactosidase ( $\beta$-gal) expression plasmid (Ambion), and equal amounts (100 pmol) of miR-143 mimic, inhibitor or scrambled negative control RNA (RiboBio, Guangzhou, China) using Lipofectamine 2000 (Invitrogen; Thermo Fisher Scientific, Inc.). The $\beta$-gal plasmid was used as a transfection control. Cells were harvested at $48 \mathrm{~h}$ post-transfection and analyzed for luciferase activity using a luciferase assay kit (Promega, Madison, WI, USA).

Western blot analysis. To perform protein analysis, cell lysates were harvested and measured using western blotting, as previously described (11). Protein sample (50 $\mu \mathrm{g})$ was fractionated by $12 \%$ SDS-PAGE and transferred to nitrocellulose membranes. The membranes were blocked in 5\% skimmed milk and PBS for $2 \mathrm{~h}$ and then incubated at $4^{\circ} \mathrm{C}$ overnight. The following antibodies were used: Anti-CIAPIN1 (1:300 dilution; catalog no. ab154904; Abcam, Cambridge, UK) and anti-glyceraldehyde 3-phosphate dehydrogenase (GAPDH) antibody (1:1,000 dilution; catalog no. KC-5G4; Kangchen, Shanghai, China). GAPDH served as an internal control. After washing, the membranes were incubated with FITC-conjugated secondary antibody (1:5,000 dilution; catalog no. ab99772; Abcam) at room temperature for $1 \mathrm{~h}$. Images were captured on the Odyssey CLx Infrared Imaging System (LI-COR Biosciences, Lincoln, NE, USA). Western blot bands were quantified using Odyssey CLx v2.1 software.

Quantitative-PCR analysis. TRIzol reagent (Invitrogen; Thermo Fisher Scientific, Inc.) was used to extract total RNA according to the manufacturer's protocols. Reverse transcription (RT) was accomplished by using reverse transcriptase to synthesize cDNA. RT was performed using 10X RT buffer, Multiscribe Reverse Transcriptase, $10 \mathrm{mM}$ dNTP and RT-RNA (Roche Diagnostics GmbH, Mannheim, Germany) at $25^{\circ} \mathrm{C}$ for $10 \mathrm{~min}, 37^{\circ} \mathrm{C}$ for $2 \mathrm{~h}$ and $85^{\circ} \mathrm{C}$ for $5 \mathrm{~min}$. PCR was perfomed using 10X PCR buffer, $10 \mathrm{mM}$ dNTP, Taq enzyme and primers from the Roche SYBR Green PCR Master Mix 
A

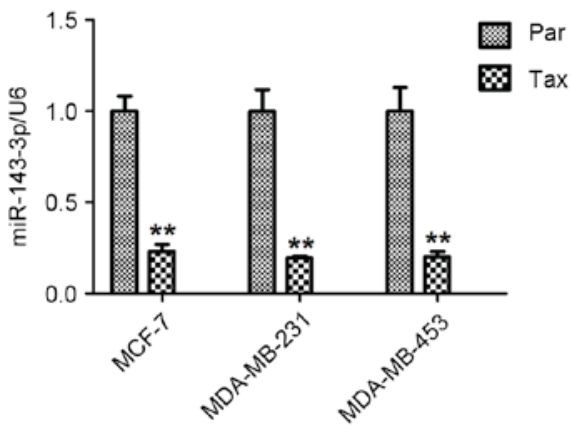

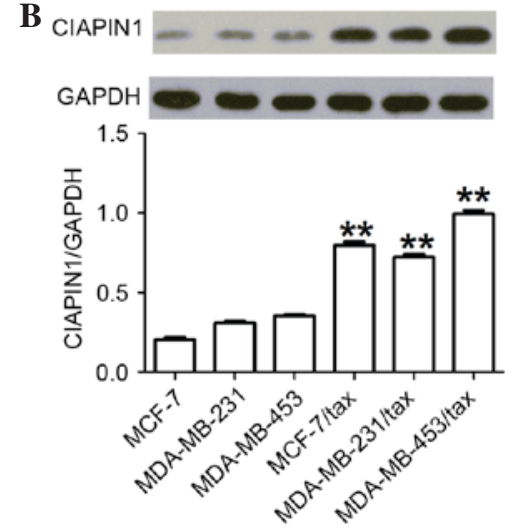

Figure 2. Expression of miR-143 and CIAPIN1 in breast cancer cell lines using quantitative-polymerase chain reaction and western blot analysis. (A and B) A significant difference is present in the expression levels of miR-143 and CIAPIN1 between MDR and non-MDR breast cancer cells. ${ }^{* *} \mathrm{P}<0.01$ for MCF7/Tax versus MCF7, MDA-MB-231/Tax versus MDA-MB-231, and MDA-MB-453/Tax versus MDA-MB-453. CIAPIN1, cytokine-induced apoptosis inhibitor 1; miR-143, microRNA-143; MDR, multidrug resistant; Tax, Taxol; GAPDH, glyceraldehyde 3-phosphate dehydrogenase; Par, non-MDR breast cancer cells or normal breast cancer cells.

kit. The cycling conditions were as follows: $95^{\circ} \mathrm{C}$ for $10 \mathrm{~min}$, $95^{\circ} \mathrm{C}$ for $15 \mathrm{sec}$ and $60^{\circ} \mathrm{C}$ for $15 \mathrm{sec}$, and $72^{\circ} \mathrm{C}$ for $15 \mathrm{sec}$, for 40 cycles. The levels of miR-143 mRNA were measured using SYBR Green incorporation on a Roche LightCycler480 Real Time PCR system (Roche Diagnostics GmbH), with U6 as an internal control. The PCR primers used were as follows: hsa-miR-143-3p forward, 5'-UGAGAUGAAGCACUGUAG CUC-3' and reverse, 5'-GTCGTATCCAGTGCGTGTCGTG-3'; and U6 forward, 5'-GCTTCGGCACATATACTAAAAT-3' and reverse, 5'-CGCTTCACGAATTTGCGTGTCAT-3'. To obtain a relative quantitation measurement, the data was analyzed using the quantification cycle $(\mathrm{Cq})$ value compared with U6 mRNA. The amount of target was determined by normalizing to endogenous reference [using the $2^{-\Delta \Delta \mathrm{Cq}}$ method (17)] and was relative to a calibrator (mean of the control samples).

Statistical analysis. Data analysis was performed using SPSS 14.0 software (SPSS Inc., Chicago, IL, USA) and Graphpad software (GraphPad Software, Inc., La Jolla, CA, USA). The data for the in vitro experiments were repeated three times and statistical comparisons between two groups were performed by t-test, and among multiple groups were performed by one-way ANOVA followed by Tukey's multiple comparison tests. Data are presented as the mean \pm standard error of the mean. Results with $\mathrm{P}<0.05$ were considered statistically significant.

\section{Results}

$T D R$. The $\mathrm{IC}_{50}$ values of MCF-7, MDA-MB-231 and MDA-MB-453 cells exposed to Taxol were measured, and it was observed that the values were significantly increased (all $\mathrm{P}<0.01$ ) (Fig. 1), indicating a significant increase in drug resistance.

Upregulation of CIAPIN1 protein and downregulation of miR-143 in TDR breast cancer cells. First, the expression of miR-143 in TDR human breast cancer cells was examined and it was found that the levels were significantly decreased (all $\mathrm{P}<0.01$ ) (Fig. 2A). Next, the study investigated the differences

\section{hsa-miR-143 3'-UTR-CIAPIN1-WT 3'-UTR-CIAPIN1-MT 3'-CUCGAUGUCACGAAGUAGAGU-5'
5'-CCUGCCUUGAGUCUCAUCUCA-3'
5'-CCUGCCUUGAGUCAATCCTCT-3'}

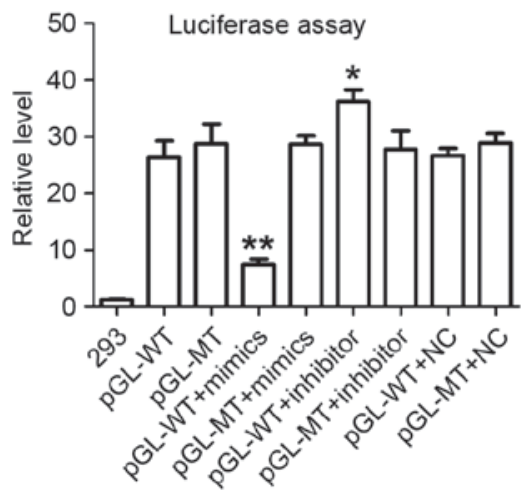

Figure 3. miR-143 downregulates CIAPIN1 by targeting its 3'-UTR. Schematic representation of the predicted target site of miR-143 in the WT and MT 3'-UTR of CIAPIN1. The seed-matching sequence is indicated. miR-143 mimic inhibited WT, but not CIAPIN1 3'-UTR reporter activity in HEK293 cells. ${ }^{* *} \mathrm{P}<0.01$ vs. pGL-WT. miR-143 inhibitor increased luciferase activity in HEK293 cells compared with the negative control group. ${ }^{*} \mathrm{P}<0.05$ vs. pGL-WT. CIAPIN1, cytokine-induced apoptosis inhibitor 1; miR-143, microRNA-143; Tax, Taxol; WT, wild-type; MT, mutant; UTR, untranslated region; pGL, pGL3-promoter vector.

in CIAPIN1 levels in various TDR breast cancer cell lines. The amount of CIAPIN1 protein was analyzed using western blotting, and a significant increase in CIAPIN1 protein levels was found in the TDR cells compared with the non-TDR cells (all $\mathrm{P}<0.01$ ) (Fig. 2B).

miR-143 targeting of the CIAPIN1 3'-UTR. Two independent informatics software packages (TargetScan and miRanda; http://www.targetscan.org and http://www.microrna.org) were used to perform a comprehensive survey for target genes according to consensus miR-143 binding sites. A sequence was found to be located at bases 373-380 of the CIAPIN1 3'-UTR (NM_020313.2), which was highly complementary with the seed sequence of miR-143 (Fig. 3). A luciferase assay was 
A
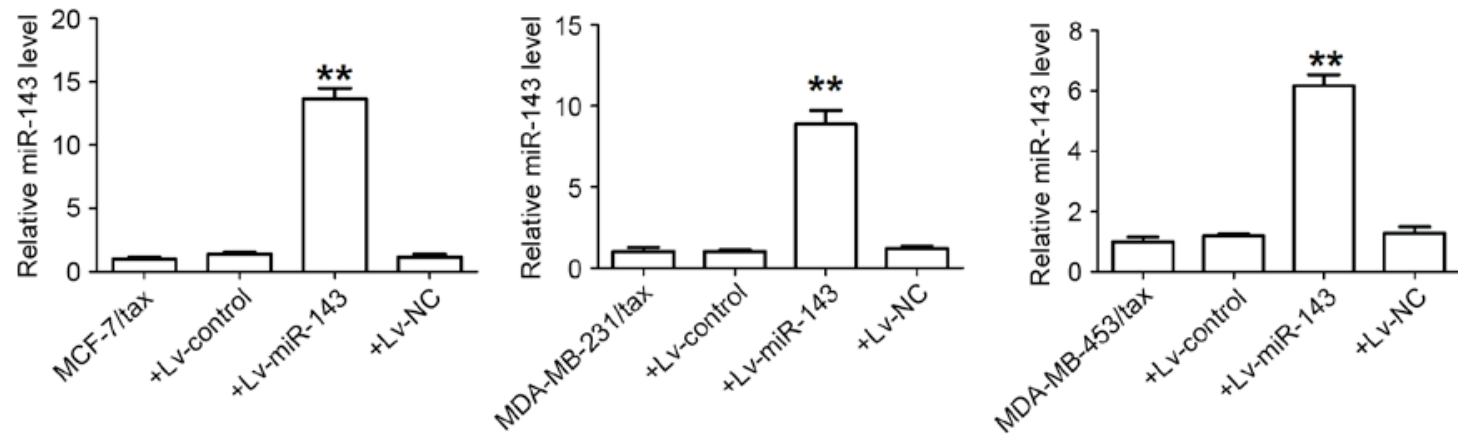

B

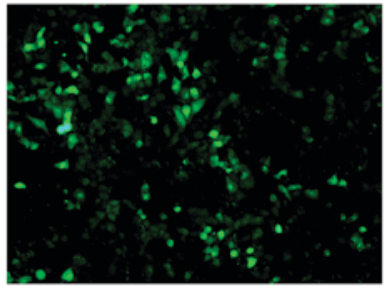

MCF-7/tax

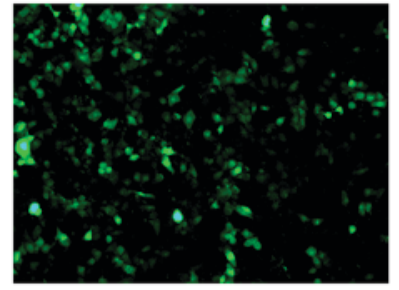

MDA-MB-231/tax

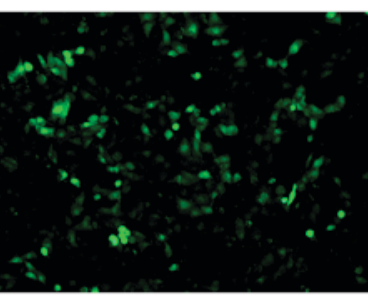

MDA-MB-453/tax

C
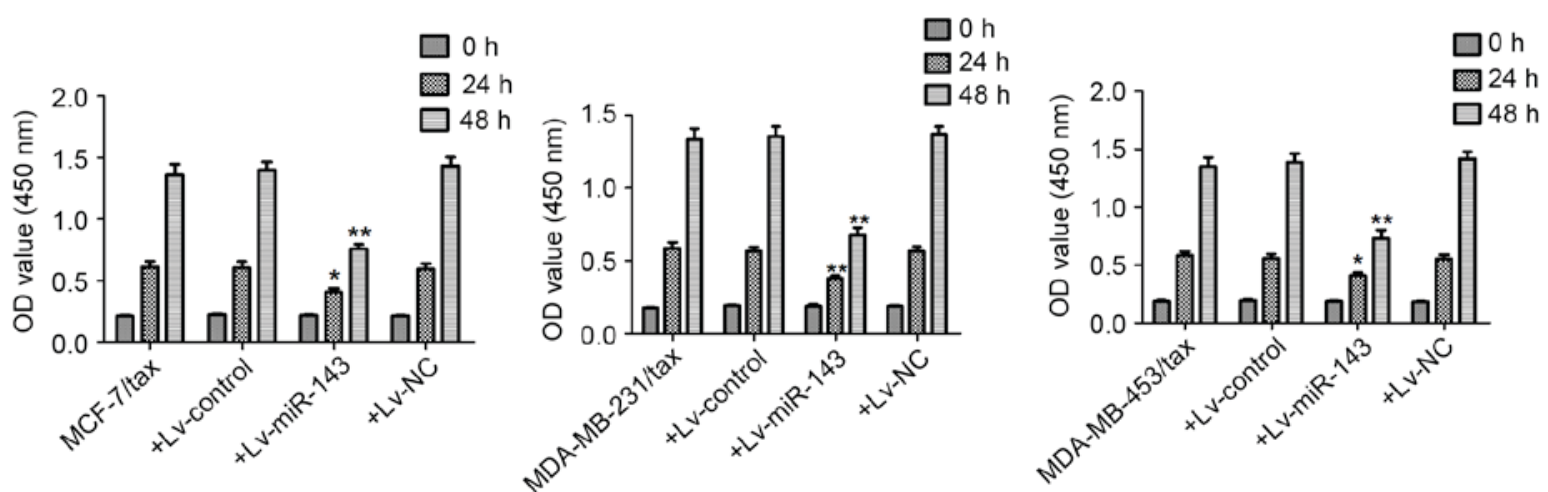

Figure 4. miR-143 attenuates the growth of breast cancer TDR cells. (A) Transcript level of miR-143 determined using quantitative polymerase chain reaction. ${ }^{* *} \mathrm{P}<0.01$ for MCF7/Tax+Lv-miR-143 versus MCF7/Tax, MDA-MB-231/Tax+Lv-miR-143 versus MDA-MB-231/Tax, and MDA-MB-453/Tax+Lv-miR-143 versus MDA-MB-453/Tax. (B) Fluorescence microscopy results of MCF7/Tax, MDA-MB-231/Tax and MDA-MB-453/Tax 96 h after lentiviral-miR-143 infection $(\mathrm{MOI}=20 \mathrm{x} 120)$. The condition of the lentiviral-miR-143 infected cells was good and the percentage of cells expressing green fluorescent protein was greater than $90 \%$ (magnification, $\mathrm{x} 120$ ). (C) miR-143 significantly attenuated cell proliferation of MCF-7, MDA-MB-231 and MDA-MB-453 TDR cells in vitro. ${ }^{*} \mathrm{P}<0.05$ for MCF7/Tax+Lv-miR-143 versus MCF7/Tax (24h), MDA-MB-453/Tax+Lv- miR-143 versus MDA-MB-453/Tax (24h), and MDA-MB-231/Tax+Lv-miR-143 versus MDA-MB-231/Tax (24h). ${ }^{* *} \mathrm{P}<0.01$ for MCF7/Tax+Lv-miR-143 versus MCF7/Tax (48h), MDA-MB-231/Tax+Lv-miR-143 versus MDA-MB-231/Tax (48h) and MDA-MB-453/Tax+Lv-miR-143 versus MDA-MB-453/Tax (48h). miR-143, microRNA-143; Tax, Taxol; TDR, Taxol-induced drug-resistant; Lv, lentivirus.

A
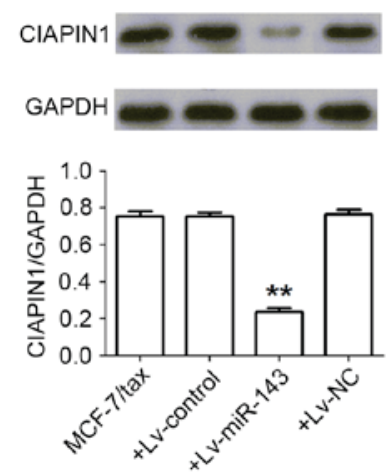

B
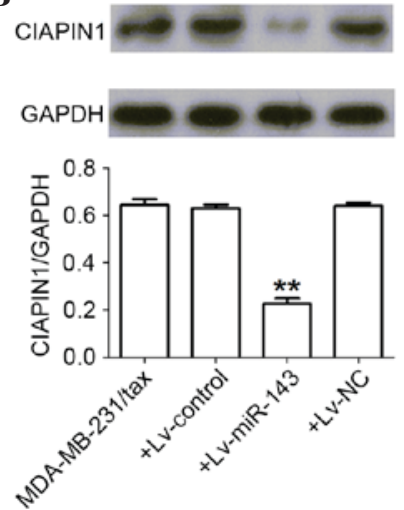

C
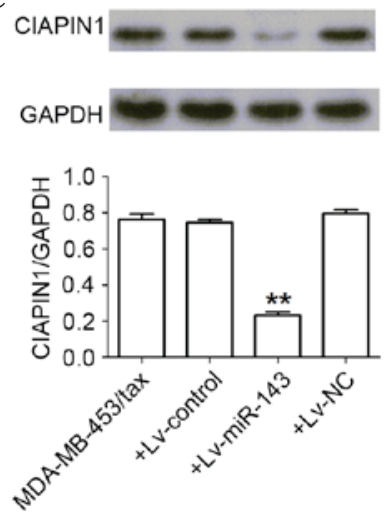

Figure 5. CIAPIN1 protein levels are decreased in breast cancer TDR cells with miR-143. Representative western blotting bands of CIAPIN1 protein for the (A) MCF-7, (B) MDA-MB-231 and (C) MDA-MB-453 cell lines. ${ }^{* *} \mathrm{P}<0.01$ for MCF7/Tax+Lv-miR-143 versus MCF7/Tax, MDA-MB-231/Tax+Lv-miR-143 versus MDA-MB-231/Tax, and MDA-MB-453/Tax+Lv-miR-143 versus MDA-MB-453/Tax. CIAPIN1, cytokine-induced apoptosis inhibitor 1; miR-143, microRNA-143; Tax, Taxol; NC, negative control; Lv, lentivirus; GAPDH, glyceraldehyde 3-phosphate dehydrogenase. 
performed to experimentally validate this target. In the presence of miR-143, the relative luciferase activity of HEK-293 cells with the wild-type (WT) construct was significantly reduced. However, cells transfected with the MT construct did not exhibit a significant suppressive effect in the presence of miR-143 (Fig. 3). In addition, the knockdown of miR-143 with a miR-143 inhibitor increased luciferase activity in WT HEK-293 cells. These results suggest a direct and specific interaction of miR-143 with the CIAPIN1 3'-UTR in HEK-293 cells. Taken together, the data suggest that CIAPIN1 is a target gene of miR-143.

Lv-miR-143 suppresses proliferation of breast cancer MDR cells. First, the expression levels of miR-143 were measured to evaluate the effectiveness of Lv-miR-143 in the MCF-7, MDA-MB-231 and MDA-MB-453 TDR cells. As expected, following transfection of Lv-miR-143, the expression of miR-143 mRNA levels was significantly increased in the MCF-7, MDA-MB-231 and MDA-MB-453 TDR cells (Fig. 4A). Lentiviral-expressed vector miR-143 was infected into the MCF-7, MDA-MB-231 and MDA-MB-453 TDR cells (MOI=20, best MOI) and a stably expressed cell line was obtained subsequent to screening (Fig. 4B). The expression of GFP and miR-143 was observed, demonstrating that the MCF-7, MDA-MB-231 and MDA-MB-453 MDR LV-miR-143 cells were of good quality (Fig. 4B). At 24 and $48 \mathrm{~h}$ after Lv-miR-143 transfection, Lv-miR-143 was found to have decreased the proliferation of the MCF-7, MDA-MB-231, and MDA-MB-453 TDR cells (Fig. 4C).

miR-143 represses CIAPIN1 expression in breast cancer $T D R$ cells. The aforementioned miRNA databases predicted that CIAPIN1 contains a putative binding site for miR-143, suggesting that it is a potential target for miR-143. Consistent with this prediction, the present results confirmed that miR-143 post-transcriptionally repressed CIAPIN1 expression by binding to the CIAPIN1 3'-UTR (P<0.01) (Fig. 5).

\section{Discussion}

MDR remains a major obstacle to achieving curative breast cancer chemotherapy treatment, and causes $>90 \%$ of metastatic breast cancer patients to experience treatment failure (18). For this reason, the development of novel avenues for overcoming MDR is urgent. MDR in breast cancer involves multiple genes and mechanisms. Current cancer research has become increasingly focused on gene therapy, and the present study aimed to improve the effectiveness of chemotherapy treatment by targeting the genes associated with breast cancer MDR. Shibayama et al (7) first identified CIAPIN1, a novel apoptosis inhibitor, in 2004. More recently, studies of MDR in gastric cancer, leukemia cell lines and breast cancer cells (8-11) have provided evidence that CIAPIN1 plays a role in the MDR process in malignant cancer. Thus, CIAPIN1 is a potential target for the improvement of therapeutic efficacy. In a study using nude mice with MDR breast cancer tumors, the combination of chemotherapy with the knockdown of CIAPIN1 levels using lentivirus-vector-based RNAi significantly inhibited tumor growth in vivo (19). In agreement with these previous studies, the present results demonstrated that
CIAPIN1 protein levels significantly increased in TDR breast cancer cells.

In numerous human tumor types, including prostate cancer, hepatocellular carcinoma, non-small cell lung cancer and gastric cancer, miR-143 is significantly dysregulated $(20,21)$. However, the role of miR-143 in breast cancer remains relatively uninvestigated. In the present study, it was found that miR-143 mRNA levels were significantly decreased in the TDR breast cancer cells. The association between miR-143 and CIAPIN1 in MCF-7, MDA-MB-231 and MDA-MB-453 TDR cells was also investigated. Bioinformatic methods were used to identify whether CIAPIN1 is a target gene of miR-143. As predicted, in the CIAPIN1 3'UTR, a highly complementary potential binding site of miR-143 was found at the bases of the CIAPIN1 3'UTR (NM_020313.2). Luciferase activity assay showed that the expression of a reporter vector containing the WT sequence of the CIAPIN1 3'UTR was inhibited by ectopic expression of miR-143. In addition, after transfecting the miR-143 mimic into TDR breast cancer cells, functional assays were performed. It was found that the expression levels of miR-143 increased, whereas the protein levels of CIAPIN1 decreased in the MCF-7, MDA-MB-231 and MDA-MB-453 TDR cells. These findings demonstrate that miR-143 functions as a CIAPIN1 suppressor. Abnormal growth is a fundamental characteristic of tumor cells, and the main reason for uncontrolled growth is due to an imbalance in cell cycle regulation (11). Experiments on the cell proliferation inhibition of tumor cells revealed that Lv-miR-143 decreases the proliferation of MCF-7, MDA-MB-231 and MDA-MB-453 TDR cells.

Together, these findings demonstrate that miR-143 participates in the reversal of TDR in human breast cancer cells by inhibiting CIAPIN1, and that it suppresses the growth of breast cancer TDR cells. These data provide compelling evidence for making the miR-143/CIAPIN1 pathway a novel target for breast cancer therapy.

\section{Acknowledgements}

This study was supported by the Natural Science Foundation of Heilongjiang Province of China (grant no. H201391).

\section{References}

1. Guo P, Huang ZL, Yu P and Li K: Trends in cancer mortality in China: An update. Ann Oncol 23: 2755-2762, 2012.

2. Seruga B, Hertz PC, Le LW and Tannock IF: Global drug development in cancer: A cross-sectional study of clinical trial registries. Ann Oncol 21: 895-900, 2010.

3. Marquette $\mathrm{C}$ and Nabell L: Chemotherapy-resistant metastatic breast cancer. Curr Treat Options Oncol 13: 263-275, 2012.

4. Gonzalez-Angulo AM, Morales-Vasquez F and Hortobagyi GN: Overview of resistance to systemic therapy in patients with breast cancer. Adv Exp Med Biol 608: 1-22, 2007.

5. Kuo MT: Roles of multidrug resistance genes in breast cancer chemoresistance. Adv Exp Med Biol 608: 23- 30, 2007.

6. Cascorbi I: Role of pharmacogenetics of ATP-binding cassette transporters in the pharmacokinetics of drugs. Pharmacol Ther 112: 457-473, 2006.

7. Shibayama H, Takai E, Matsumura I, Kouno M, Morii E, Kitamura Y, Takeda $\mathrm{J}$ and Kanakura Y: Identification of a cytokine-induced antiapoptotic molecule anamorsin essential for definitive hematopoiesis. J Exp Med 199: 581-592, 2004.

8. Hao Z, Li X, Qiao T, Du R, Hong L and Fan D: CIAPIN1 confers multidrug resistance by upregulating the expression of MDR-1 and MRP-1 in gastric cancer cells. Cancer Biol Ther 5: 261-266, 2006. 
9. Li X, Fan R, Zou X, Hong L, Gao L, Jin H, Du R, He L, Xia L and Fan D: Reversal of multidrug resistance of gastric cancer cells by down-regulation of CIAPIN1 with CIAPIN1 siRNA. Mol Biol (Mosk) 42: 102-109, 2008 (In Russian).

10. Li X, Hong L, Zhao Y, Jin H, Fan R, Du R, Xia L, Luo G and Fan D: A new apoptosis inhibitor, CIAPIN1 (cytokine-induced apoptosis inhibitor1), mediates multidrug resistance in leukemia cells by regulating MDR-1, Bcl-2, and Bax. Biochem Cell Biol 85: 741-750, 2007.

11. Lu D, Xiao Z, Wang W, Xu Y, Gao S, Deng L, He W, Yang Y, Guo $X$ and Wang X: Down regulation of CIAPIN1 reverses multidrug resistance in human breast cancer cells by inhibiting MDR1. Molecules 17: 7595-7611, 2012.

12. $\mathrm{Li} \mathrm{X}, \mathrm{Wu} \mathrm{K}$ and Fan D: CIAPIN1 as a therapeutic target in cancer. Expert Opin Ther Targets 14: 603-610, 2010.

13. Andorfer CA, Necela BM, Thompson EA and Perez EA: MicroRNA signatures: Clinical biomarkers for the diagnosis and treatment of breast cancer. Trends Mol Med 17: 313-319, 2011.

14. Shi M and Guo N: MicroRNA expression and its implications for the diagnosis and therapeutic strategies of breast cancer. Cancer Treat Rev 35: 328-334, 2009.

15. Jiang S, Zhang LF, Zhang HW, Hu S, Lu MH, Liang S, Li B, Li Y, Li D, Wang ED and Liu MF: A novel miR-155/miR-143 cascade controls glycolysis by regulating hexokinase 2 in breast cancer cells. EMBO J 31: 1985-1998, 2012.
16. Yan X, Chen X, Liang H, Deng T, Chen W, Zhang S, Liu M, Gao X, Liu Y, Zhao C, et al: miR-143 and miR-145 synergistically regulate ERBB3 to suppress cell proliferation and invasion in breast cancer. Mol Cancer 13: 220, 2014.

17. Livak and Schmittgen: Analysis of relative gene expression data using real-time quantitative PCR and the $2-\Delta \Delta \mathrm{Ct}$ method. Methods 25: 402-408, 2001.

18. Egerton N: Ixabepilone (ixempra), a therapeutic option for locally advanced or metastatic breast cancer. P T 33: 523-531, 2008.

19. Wang XM, Gao SJ, Guo XF, Sun WJ, Yan ZQ, Wang WX, Xu YQ and Lu D: CIAPIN1 gene silencing enhances chemosensitivity in a drug-resistant animal model in vivo. Braz J Med Biol Res 47: 273-278, 2014.

20. Zhang ZQ, Meng H, Wang N, Liang LN, Liu LN, Lu SM and Luan Y: Serum microRNA 143 and microRNA 215 as potential biomarkers for the diagnosis of chronic hepatitis and hepatocellular carcinoma. Diagn Pathol 9: 135, 2014.

21. Zhuang M, Shi Q, Zhang X, Ding Y, Shan L, Shan X, Qian J, Zhou X, Huang Z, Zhu W, et al: Involvement of miR-143 in cisplatin resistance of gastric cancer cells via targeting IGF1R and BCL2. Tumour Biol 36: 2737-2745, 2015. 